Cipango $\begin{aligned} & \text { Cipango } \\ & \text { Cahiers d'études japonaises }\end{aligned}$

15 | 2008

Guerre, colonialisme et commémoration

\title{
L'expérience de l'art abstrait au Japon. Une nouvelle conscience de l'art à partir des années 1970
}

Marie Parra-Aledo

\author{
(2) OpenEdition \\ Journals \\ Édition électronique \\ URL : https://journals.openedition.org/cipango/444 \\ DOI : $10.4000 /$ cipango.444 \\ ISSN : 2260-7706 \\ Éditeur \\ INALCO
}

Édition imprimée

Date de publication : 1 janvier 2008

Pagination : 285-289

ISBN : 978-2-85831-177-4

ISSN : 1164-5857

Référence électronique

Marie Parra-Aledo, « L'expérience de l'art abstrait au Japon. Une nouvelle conscience de l'art à partir des années 1970 », Cipango [En ligne], 15 | 2008, mis en ligne le 14 novembre 2011, consulté le 30 juin 2021. URL : http://journals.openedition.org/cipango/444 ; DOI : https://doi.org/10.4000/cipango.444

Ce document a été généré automatiquement le 30 juin 2021.

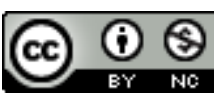

Cipango est mis à disposition selon les termes de la Licence Creative Commons Attribution - Pas d'Utilisation Commerciale 4.0 International. 


\title{
L'expérience de l'art abstrait au Japon. Une nouvelle conscience de l'art à partir des années 1970
}

\author{
Marie Parra-Aledo
}

\section{RÉFÉRENCE}

L'expérience de l'art abstrait au Japon. Une nouvelle conscience de l'art à partir des années 1970, Thèse de doctorat soutenue par Marie Parra-Aledo (Lyon 3, 2007), sous la direction de J. Chollet.

1 Une nouvelle réflexion sur la forme, notamment à partir des années 1970 au Japon, aboutit à deux constats dont nous nous sommes efforcée d'analyser le fondement. D'une part, le fait que l'image nécessite la parole écrite et que désormais la forme silencieuse vivra le plus souvent en symbiose avec l'écrit. D'autre part, que l'objet d'art n'aura désormais de valeur que s'il est pensé associé à un vécu.

2 Ce sont les productions plastique et littéraire d'un artiste, Hayashi Yoshifumi 林芳文 (1943-2001), actif dans les années 1980 au Japon, qui ont étayé nos deux hypothèses de travail. Ce sont les écrits de cet artiste que nous envisagerons comme une source historique, scientifique, philosophique, mais aussi comme une inspiration sensible permettant une analyse de l'évolution majeure que représente dans l'histoire des idées au Japon l'avènement de la pensée abstraite en art.

Le premier constat, la nécessité de l'écrit auprès de l'œuvre d'art faisait dire à René Clair que curieusement au xxe siècle, il semblait que plus l'œuvre d'art se minimisait, voire se faisait quasi invisible, plus le monument de papier qui lui était consacré était imposant. Notre second angle de vue, celui du vécu de l'objet d'art et de son créateur, s'appuie sur l'analyse d'une rencontre intellectuelle entre des artistes devenus des figures marquantes des années 1970-80, rencontre qui prit fortuitement l'appellation de «mouvement », de « groupe », connu sous le nom de Mono ha もの派, dont personne 
ne sait exactement comment il fut forgé, si ce n'est qu'il apparaît au tout début des années 1970. Les personnes concernées dans cette association ne se considérèrent jamais comme les fondateurs ni d'un groupe formel ni d'un mouvement. Cependant, le mot fut attaché à l'idée d'art " écologique » ou « d'art pauvre ».

Hayashi Yoshifumi se trouva au cœur des débats, des rencontres, des amitiés et des actions des années 1970-80. Celui-ci, écrivant, durant les deux dernières années de sa vie, une œuvre à la fois d'érudition et romanesque, apporta, nous semble-t-il, à sa famille intellectuelle, voire à sa génération, une réflexion écrite qui compense l'absence de texte théorique de ce qui fut hâtivement appelé le " mouvement » Mono ha.

L'aboutissement de sa carrière artistique, qui, sur une trentaine d'années, entre 1970 et 2000, lui fournit la matière d'une réflexion sur la forme abstraite, est un roman, une œuvre imposante de plus de quatre cents pages, intitulée Le dragon de Sessh $\bar{u}$ (Sesshū no $r y \bar{u}$ 雪舟の龍), parue aux éditions NHK en 2000. Il s'agit d'un parcours très documenté, dans le monde des arts au Japon.

Hayashi, qui montrait régulièrement ses travaux dans les galeries de Tōkyō, nous fait découvrir le monde de l'art tel qu'un artiste le perçoit dans les années 1980. Nous suivons dans ce roman un personnage souhaitant authentifier un tableau trouvé de façon inopinée chez lui. Le tableau rappelant fortement le style de Sesshū, maître du dessin à l'encre du XvI ${ }^{e}$ siècle, le propriétaire se décide à le faire expertiser et fait appel pour ce faire à un artiste, l'auteur lui-même.

7 Hayashi est né à Ōsaka le 13 juillet 1943 de parents coréens émigrés au Japon. Un fait douloureux marque sa jeunesse. L'une de ses plus jeunes sœurs est enlevée encore enfant. On ne la retrouvera jamais. Hayashi évoquait souvent le souvenir douloureux de ce drame familial, inexorablement lié à l'arrivée de ses parents au Japon, le pays d'accueil qui leur vola un enfant. Admis à l'université Waseda, en Littérature, Hayashi dut interrompre ses études lorsque son père décéda, pour s'occuper de ses jeunes sœurs. Hayashi se marie en 1978. Ses beaux-parents dirigeaient une entreprise de copie de tableaux où leur fille était employée à reproduire des tableaux de maîtres japonais. En décembre 1997, l'un de ses deux fils, Nobumasa 伸優, âgé de dix-huit ans, se suicide. Ce drame afflige profondément Hayashi. Après vingt-cinq ans d'expositions régulières dans de nombreuses galeries, il se consacra à l'écriture dont nous pensons qu'elle le situe au rang de véritable penseur de l'art japonais de la fin du $\mathrm{xx}^{\mathrm{e}}$ siècle. Le 22 août 2001, peu après la publication de son ouvrage, il se suicida à son tour.

Hayashi s'ouvrit à l'art international alors qu'il était employé par la galerie Nantenshi, d'abord à Ōsaka en 1973 et l'année suivante à Tōkyō où la galerie avait une succursale. Ses rencontres lui permirent d'approfondir une réflexion fondée sur une solide culture classique où il posa la question, non sans humour, du rapport de l'artiste aux marchands, aux experts, aux critiques, aux membres des universités et des musées, mais surtout au réel, au temps et à la pensée abstraite. Un des thèmes récurrents dans sa réflexion est la façon dont l'art abstrait s'est imposé au Japon, aux dépens des supports et des cadres conventionnels dans lesquels évoluait la représentation de la forme jusque-là.

9 Quant à cette rencontre à laquelle certains critiques occidentaux, ignorant souvent tout de la langue et de la culture japonaises, eurent tôt fait d'accoler le mot " groupe » et le nom Mono ha, elle fut bien une rencontre intellectuelle entre quelques artistes marquants des trois dernières décennies du vingtième siècle. Le sculpteur Sekine 
Nobuo 関根伸夫, Hayashi Yoshifumi, son ami et collaborateur, le peintre Lee U Fan 李禹 煥, entre autres. Ce dernier expliquait très bien la force de cette amitié dans un texte synthétique très clair où il évoquait l'étonnement de ses amis devant ce "mouvement " dont on leur attribuait la paternité et où il écrit que «le nom semblait être surgi de nulle part».

Cependant, Mono ha fut le paradigme d'un certain type d'actions menées par des artistes actifs dans les années 1960-70, davantage qu'à un certain type d'objets, actions qui se caractérisaient par l'utilisation de matériaux récupérés. L'intention était de questionner l'art occidental et ses traditions dont on estimait avoir littéralement " hérité au Japon », l'expression étant du critique et professeur Minemura Toshiaki 峯 村敏明. Au-delà, il s'agissait de défier les arts conventionnels, bien qu'aucune déclaration formelle écrite ne scellât cette volonté.

11 Le nom même dudit mouvement, constitué de l'homophone du mot objet, laisse flotter un doute sur le fait qu'il s'agit de l'objet matériel lui-même ou de l'intention de se projeter dans un objet idéel. Nom et mot se confondant à l'extrême. Sawaragi Noi 椹木野 衣 dans son ouvrage Japon Modernité · Arts (Nihon·Gendai B Bijutsu) consacre quelques pages à cette homophonie, (inconsciente selon nous), entre les deux mots objet qui se prononcent tous deux mono. Celui propre à la littérature, bien connu dans l'expression mono no aware, qui ne s'écrit pas avec un idéogramme mais phonétiquement, serait une façon provocatrice de poser la question de ce qui est artificiellement importé et non créé historiquement au Japon. Les nombreuses expressions proposées pour traduire le mot attestent de la richesse de l'expression. Pour Kawabata Yasunari, il s'agit de « l'étrange tristesse des choses », pour Alain Walter de « la sensation fugitive de la beauté et de l'être d'une chose », pour Jacqueline Pigeot de «la touchante fragilité des êtres", pour ne citer que ces quelques exemples parmi de nombreuses autres approches.

Mono ha sera l'expression d'une révolte des artistes contre l'objet d'art en tant qu'objet monnayable, auquel est attachée une valeur marchande plus ou moins abstraite au départ, destinée à évoluer avec le temps. L'objet doté d'une valeur marchande dans un temps mesurable est nié parce que le temps économique, l'homme économique sont niés. Désormais, tout objet, appréhendé comme faisant partie du temps sensible, du temps psychique, n'est reconnu que comme essentiellement lié à et porteur d'un vécu.

Toute la question de la pérennité de l'œuvre d'art se trouve ainsi également posée dans le fait de la marchandisation de l'art et la question de l'authentification et de la reconnaissance d'un l'objet est bien aussi au centre du roman de Hayashi, question qui donna lieu par ailleurs, à partir de l'ouvrage de Hayashi, à une série de documents culturels télévisés.

14 Hayashi Yoshifumi, collaborateur privilégié de Sekine, dont le critique Mizuma Toshitaka 水間敏隆, directeur artistique de la galerie Bijutsu sekai à Tōkyō, jetant un regard rétrospectif sur les textes que Hayashi avaient écrits vingt-cinq ans plus tôt, largement influencé par les progrès des relations entre le Japon et ses voisins asiatiques, dont la Corée, disait qu'il voyait en lui un artiste d'une rare clairvoyance. Mizuma, commentant une exposition de Sekine à Tōkyō en 2005, écrit : «Alors que l'on pense généralement que le mouvement Mono ha est né au Japon, il faut y voir l'aboutissement d'une sagesse qui transcende les frontières même du Japon et de la Corée. Après s'être assuré une base idéologique, Mono ha a continué à se réaliser dans l'esprit du relativisme [sōtaishugi 相対主義] qui caractérise la pensée orientale, 
influençant même les points de vue monistes [ichigenron 一元論] de la pensée occidentale ».

15 Hayashi Yoshifumi se situe au confluent des noms marquant l'évolution des idées au Japon dans les années 1970, Lee U Fan, Sekine Nobuo, Yokoo Tadanori 横尾忠則, le critique d'art Takahashi Masayuki 高橋雅之, Yoshida Makoto 吉田誠, Kaku Jinshoku 郭仡 植, Ishiko Junzō 石子順造 et bien d'autres. Il éprouva la nécessité, comme une urgence, d'un travail théorique sur le thème d'une nouvelle forme de la pensée, à partir de l'avènement de la pensée abstraite. S'il présenta cette réflexion philosophique dans un style romancée, il n'en demeure pas moins qu'elle porte et éclaire les grandes questions du rapport de l'art au temps et au réel. 